\title{
TSOL18 vaccine antigen of Taenia solium: development of monoclonal antibodies and field testing of the vaccine in Cameroon
}

\section{Emmanuel Assana}

Supervisor: Pierre Dorny (1)

Co-supervisors: Stanny Geerts (2), Marshall W. Lightowlers (3), André P. Zoli (4)

(I) Department of Virology, Parasitology and Immunology, Ghent University

(2) Institute of Tropical Medicine, Antwerp

(3) University of Melbourne, Australia

(4) University of Dschang, Cameroon

Public defence: 29 June 2010

Taenia solium is a taeniid tapeworm, transmitted between humans and pigs. This zoonotic parasite has been eradicated in industrialized countries but is still very prevalent in developing countries. Both the adult and larval stages can establish in humans, causing taeniasis and cysticercosis respectively. Cysticerci may lodge in the brain and cause neurocysticercosis, one of the most important causes of acquired epilepsy in endemic areas. Control of T. solium is based on improvement of sanitation and pig husbandry systems, meat inspection and mass treatment of humans. Novel approaches include anthelmintic treatment and vaccination of pigs. The recombinant oncospheral antigen TSOLI8 is the most promising vaccine candidate and has shown near $100 \%$ efficacy in experimental conditions. This thesis aimed to testing the vaccine in the field and to developing techniques to assess the success of vaccination.

Chapter I reviews the literature on the immunological aspects of taeniid cestode infections and the existing vaccines against T. solium cysticercosis in pigs. In chapter 2 an epidemiological study on porcine cysticercosis is described in the far north region of Cameroon, where a vaccine field trial with TSOLI8 was going to be organized. Risk factors for transmission of T. solium were present and serological analysis of pigs showed a prevalence of porcine cysticercosis of $26.6 \%$. Chapter 3 reports on studies to characterize whether the principal antibody specificities raised by TSOLI 8 in pigs are against linear or conformational determinants. The original TSOLI8 protein and two truncations were used in inhibition ELISA to determine their ability to inhibit the binding of protective pig antibodies to TSOLI8. The results indicated that the dominant antibody specificities, and likely the host protective specificities, of TSOLI8 are conformational epitopes. Chapter 4 describes the development of an antibody detection test for the specific diagnosis of porcine cysticercosis. A fraction with a major band of $\mathrm{I}_{4} \mathrm{kDa}$ was obtained from crude cyst fluid of T. solium cysticerci by 2-step chromatography. In chapter 5 the efficacy of the TSOLI8 vaccine is assessed under field conditions in Cameroon. Two hundred and forty 2-3 month old piglets were involved in the study. One or more pairs of piglets were 
assigned to households, with one animal of each pair being vaccinated and the other acting as a non-vaccinated control. Vaccinated animals received three immunizations intramuscularly. At the time of the second immunization both vaccinated and control animals received an oral dose of $30 \mathrm{mg} / \mathrm{kg}$ oxfendazole. Necropsies were undertaken when the pigs were approximately 12 months of age. Viable $\mathrm{T}$. solium cysticerci were identified in 20 control pigs, including 14 animals that were massively infected. No cysticerci were found in any of the vaccinated animals indicating that the vaccine provided a very high level of protection against naturally acquired infection with $T$. solium in pigs. In chapter 6, a monoclonal antibody (MoAb)-based inhibitive ELISA was developed to assess immune responses in vaccinated pigs that correlate with protection. In all vaccinated pigs screened at necropsy, anti-TSOLI8 antibodies inhibited the binding of a MoAb specific to the conformational epitopes of TSOLI8 antigen, suggesting an immune response that correlates with protection. In chapter 7 the efficacy of the TSOLI 8 vaccine is compared with that of other vaccines. Future research activities are suggested to improve our knowledge on the duration of the immunity of the vaccine and various other aspects of the vaccine production and delivery. The combined application of TSOLI8 vaccination and a single oxfendazole treatment in pigs is a simple and relatively sustainable procedure that has the potential to control T. solium transmission in endemic areas and, indirectly, reduce the number of new cases of neurocysticercosis in humans.

\section{References}

Assana, E., Kanobana, K., Tume, C., Zoli P.A, Nguekam, Geerts, S., Berkvens, D., Dorny, P. (2007). Isolation of a $14 \mathrm{KDa}$ antigen from Taenia solium cyst fluid by HPLC and its evaluation in enzyme linked immunosorbent assay for diagnosis of porcine cysticercosis. Research in Veterinary Science 82: 370-376.

Assana, E., Amadou, F., Thys, E., Lightowlers M.W., Zoli A.P., Dorny, P., Geerts, S. (2010). Pig-farming systems and porcine cysticercosis in the north region of Cameroon. Journal of Helminthology 84: 44I-446.

Assana, E., Kyngdon, C. T., Gauci, C. G., Geerts, S., Dorny, P., De Deken, R., Anderson, G. A., Zoli, A.P., Lightowlers, M. W. (2010). Elimination of Taenia solium transmission to pigs in a field trial of the TSOL18 vaccine in Cameroon. International Journal for Parasitology, 40: 515-519.

Assana, E., Gauci, C.G., Kyngdon, C., Geerts, S, Dorny, P., Zoli, A.P., Lightowlers, M.W. (2010). Antibody responses to the host-protective Taenia solium oncosphere protein TSOL18 in pigs are directed against conformational epitopes. Parasite Immunology 32: 399-405. 